\title{
Attempts to Eradicate Methicillin-Resistant Staphylococcus aureus From a Long-Term-Care Facility With the Use of Mupirocin Ointment
}

\author{
Carol A. Kauffman, M.D., Margaret S. Terpenning, M.D., Xiaogong He, M.D., \\ LIDIJA T. ZARINS, M.S., MARY A. RAMSEY, B.S., KAREN A. JORGENSEN, R.N., Ann Arbor, Michigan, \\ WILlIAM S. SOTTILE, Ph.D., Houghton, Michigan, SUZANNE F. BRADLEY, M.D., Ann Arbor, Michigan
}

PURPOSE: To assess the impact of the use of mupirocin ointment on colonization, transmission, and infection with methicillin-resistant Staphylococcus aureus (MRSA) in a long-termcare facility.

PATIENTS AND METHODS: All 321 residents of a Veterans Affairs long-term-care facility from June 1990 through June 1991 were studied for MRSA colonization and infection. MRSA-colonized patients received mupirocin ointment to nares in the first 7 months and to nares and wounds in the second 5 months. The effect of mupirocin use on MRSA colonization and infection was monitored. All S. aureus strains isolated were tested for the development of resistance to mupirocin.

RESULTS: A total of 65 patients colonized with MRSA received mupirocin ointment. Mupirocin rapidly eliminated MRSA at the sites treated in most patients by the end of 1 week. Weekly maintenance mupirocin was not adequate to prevent recurrences- $40 \%$ of patients had recurrence of MRSA. Overall, MRSA colonization in the facility, which was $22.7 \% \pm 1 \%$ prior to the use of mupirocin, did not change when mupirocin was used in nares only $(22.2 \% \pm 2.1 \%)$, but did decrease to $11.5 \% \pm 1.8 \%$ when mupirocin was used in nares and wounds. Although colonization decreased, roommate-to-roommate transmission and MRSA infection rates, low to begin with, did not change when mupirocin was used. Mupirocin-resistant MRSA strains were isolated in $10.8 \%$ of patients.

From the Divisions of Infectious Diseases and Geriatrics (CAK, MST, XH, LTZ, MAR, KAJ, SFB), Department of Internal Medicine, Department of Veterans Affairs Medical Center. University of Michigan Medical School, Ann Arbor, Michigan, and the Michigan Department of Public Health (WSS), Houghton, Michigan.

This study was supported by a Department of Veterans Affairs Health Services Research and Development Award.

Requests for reprints should be addressed to Suzanne F. Bradley, M.D.. Department of Veterans Affairs Medical Center, 2215 Fuller Road, Ann Arbor, Michigan 48105.

Manuscript submitted April 30, 1992, and accepted in revised form September 29, 1992.
CONCLUSIONS: Mupirocin ointment is effective at decreasing colonization with MRSA. However, constant surveillance was required to identify patients colonized at admisaion or experiencing recurrence of MRSA during maintenance treatment. Long-term use of mupirocin selected for mupirocin-resistant MRSA strains. Mupirocin should be saved for use in outbreak situations, and not used over the long term in facilities with endemic MRSA colonization.

Colonization and infection with methicillin-resistant Staphylococcus aureus (MRSA) have become major concerns over the last decade in both acute- and chronic-care settings [1-9]. While some studies have documented that long-term-care facilities serve as a reservoir for MRSA and contribute to the spread of the organism when patients are transferred to acute-care hospitals [4], other studies have shown the important role that hospitalized patients colonized with MRSA play in bringing MRSA into long-term-care facilities [7]. To date, the role of long-term-care facilities in the perpetuation and spread of MRSA has not been completely elucidated $[8,9]$.

Attempts to eradicate colonization with MRSA are frequently initiated in patients in acute-care hospitals and long-term-care facilities. One method that has been used is application of mupirocin ointment to the anterior nares. Mupirocin has excellent antistaphylococcal activity and has been shown to be effective in treating staphylococcal skin infections [10]. Eradication of staphylococci from the anterior nares with mupirocin has been shown for both health care workers and patients who are colonized with MRSA [11-14]. However, whether the use of mupirocin will decrease colonization and ultimately infections with MRSA in patients in the long-term-care setting is not known. Whether the use of mupirocin is without risks, including the development of resistance, also has not been established. 
We previously assessed the importance of MRSA in our long-term-care facility. Over the course of 1 year, we determined the prevalence and incidence of colonization, patterns of acquisition, and risk of infection with MRSA [7]. We undertook the current study to assess the effect of the use of mupirocin on colonization, transmission, and infection with MRSA in this facility and to determine whether long-term maintenance therapy with mupirocin was associated with the development of resistance to the drug.

\section{PATIENTS AND METHODS}

\section{Facility and Patients}

The Ann Arbor Veterans Affairs Nursing Home Care Unit is a 120-bed long-term-care facility attached to the 300-bed acute-care hospital. Patients are cared for by three services-geriatric evaluation, rehabilitation, and long-term care-but these three services are not physically separated.

\section{Study Design}

During the initial year (June 1989 to June 1990) of this several-year study, baseline data regarding MRSA colonization and infection were obtained on all patients in the facility. The results have been published previously [7]. During the second year of the study (June 1, 1990, to June 1, 1991), all patients colonized with MRSA were treated with mupirocin ointment in an attempt to decrease MRSA colonization and to determine whether this had an effect on infection rates.

Initial surveillance cultures were performed on all patients in the facility when the second year began (June 1990). Over the course of the next year, all patients had cultures on admission to the facility, monthly as long as they remained in the facility, and whenever they returned to the facility after transfer to the acute-care hospital. After MRSApositive patients were entered into the mupirocin study, they had surveillance cultures performed weekly until the study ended on June 1, 1991.

From mid-June 1990 to the end of December 1990 , patients colonized with MRSA in their anterior nares were treated with nasal application of mupirocin; wounds were not treated. From January 1 , 1991 , to June 1, 1991, mupirocin was used in both anterior nares and wounds.

\section{Definition of Colonization/Infection}

Patients were considered colonized with MRSA when one or more cultures from any site yielded MRSA on one or more occasions [7]. MRSA infections were defined by the criteria set forth by the Centers for Disease Control [15].

\section{Drug Regimen}

All patients who had cultures from anterior nares or wounds positive for MRSA were asked to participate in the study. The study protocol had been approved by the Human Studies Subcommittee and the Research and Development Committee of the Ann Arbor Veterans Affairs Medical Center, and all patients gave written consent.

Topical mupirocin $2 \%$ ointment in a polyethylene glycol base was applied as a thin film to anterior nares and the base of the wound by the nursing staff using a sterile swab. Mupirocin was applied to wounds at the same time each day in conjunction with a routine dressing change. Wounds were covered with fine-mesh gauze and gauze bandages, not with occlusive dressings. Anterior nares were treated daily for 1 week; wounds were treated daily for 2 weeks. After this, both sites were treated on Monday, Wednesday, and Friday for 3 weeks, and then on Wednesday thereafter. All patients were treated for a minimum of 3 months after all cultures were negative for MRSA before treatment was stopped. Weekly surveillance cultures were performed every Tuesday.

If patients remained colonized on the first followup culture (done on a day off therapy in Week 2 for nares and Week 3 for wounds), daily mupirocin treatment was continued until they were culturenegative; then the regimen outlined above was employed. When relapses with one or two colonies occurred, no change was made in the treatment regimen. If more than two colonies were isolated, daily treatment was again initiated for 2 weeks followed by once-weekly treatment after this.

Patients were asked whether they had side effects from application of mupirocin (burning, itching, congestion, pain); signs of irritation (rhinorrhea, erythema) were also assessed.

\section{Microbiologic Methods}

The following sites were assessed for MRSA colonization: anterior nares, perineum, rectum, and wounds. These areas were swabbed with sterile rayon-tipped applicator sticks, which were then placed immediately into Stuart transport media and within 2 hours streaked onto colistin nalidixic acid agar containing $5 \%$ sheep's blood. After incubation at $35^{\circ} \mathrm{C}$ for 24 hours, yellow or white colonies were selected. Catalase-positive, Accu-Staph (CarrScarborough, Decatur, GA)-positive organisms were identified as $S$. aureus. Organisms that grew after 24 hours at $35^{\circ} \mathrm{C}$ on Mueller-Hinton agar plates containing $6 \mu \mathrm{g} / \mathrm{mL}$ oxacillin and $4 \%$ sodium chloride were confirmed as MRSA; methicillin-sensitive $S$. aureus (MSSA) showed no growth on oxa- 
cillin plates [16]. All isolates were phage-typed by standard methods at the Michigan Department of Public Health Laboratory [17].

Mupirocin resistance was determined initially by a screening method utilizing growth on MuellerHinton agar containing $2 \mu \mathrm{g} / \mathrm{mL}$ mupirocin. The minimal inhibitory concentration (MIC) of mupirocin for each isolate was confirmed using a microtiter method described previously [18]. High-level resistance was defined as an MIC greater than 100 $\mu \mathrm{g} / \mathrm{mL}$, low-level resistance as an MIC between 4 $\mu \mathrm{g} / \mathrm{mL}$ and $100 \mu \mathrm{g} / \mathrm{mL}$, and sensitive as less than or equal to $2 \mu \mathrm{g} / \mathrm{mL}$.

On three separate occasions (June 1990, January 1991, and June 1991), the environment was sampled for MRSA using Rodac plates (Becton Dickinson, Lincoln Park, NJ) containing mannitol salt agar (Difco, Detroit, MI) as described previously [7]. Every occupied room was sampled at the following sites: floor beside the patient's bed, bed rail, bedside table, bathroom floor, and sink. Common areas (nursing stations, television rooms, recreation rooms) were also sampled. Sampling was performed prior to the use of mupirocin, after the first 7 months when anterior nares alone had been treated, and after the next 5 months when anterior nares and wounds had been treated. Phage typing was done on all isolates. No changes had occurred in housekeeping routines over the course of the year in which the environmental samples were taken.

\section{Statistics}

Results are expressed as mean \pm SE. The $\chi^{2}$ test or one-way analysis of variance was used to compare colonization rates.

\section{RESULTS}

Overall, 321 patients were followed from June 1, 1990, to June 1, 1991. Admission and monthly surveillance cultures were obtained on these patients, and MRSA colonization and infection rates were determined. The mean age of the patients was $65.2 \pm 0.6$ years; 315 were men and 6 were women.

\section{Mupirocin Treatment}

Eighty-nine of the 321 patients (27.7\%) were found to be colonized with MRSA and were asked to participate in the mupirocin treatment trial. Of these 89 patients, only 2 refused either mupirocin treatment or weekly surveillance cultures and remained in the facility; 12 others were transferred, discharged, or died prior to beginning therapy with mupirocin, and 10 received less than 2 weeks of therapy prior to discharge or death. These $24 \mathrm{pa}-$ tients are not included in the analysis of the efficacy of mupirocin.

Thus, a group of 65 patients (20.2\% of the 321 patients in the overall study) were treated with mupirocin and followed to assess the effects of treatment on MRSA colonization; 3 of the 65 patients were treated on 2 separate occasions either 1 month (2 patients) or 2 months (1 patient) apart. The mean age of the patients treated was $64.2 \pm 1.5$ years; there were 63 men and 2 women. The mean time they were treated with mupirocin was $3.4 \pm 0.3$ months; the mean time patients were followed with weekly cultures was $4.6 \pm 0.5$ months.

Of the 65 patients, 48 were entered into the study during the first 7 months and received mupirocin to anterior nares only. In the next 5 months, there were 17 new patients entered into the study. In addition, eight patients who had entered during the first 7 months continued in the study and two patients were treated for a second time when they were readmitted to the facility during the second 5 months.

No patient experienced local irritation from the application of mupirocin ointment, no signs of local irritation were noted, and no patient left the study because of intolerance to the drug.

\section{Eradication of MRSA Colonization in Individual Patients}

Mupirocin quickly eliminated MRSA from the anterior nares. In the first 6 months, 40 of $48 \mathrm{pa}-$ tients (83.3\%) had cleared their nares of MRSA by the end of 1 week of treatment, and 45 of 48 (93.7\%) had clearance by the end of Week 3 . In the second 5 months, when both nares and wounds were treated, MRSA was eliminated more rapidly from the nares; 18 of $19(94.7 \%)$ patients had clearance in 1 week and all had clearance by Week 3 (Figure 1).

In the first 7 months, wound MRSA colonization data were available from monthly surveillance cultures only. Treatment of anterior nares alone had minimal effects on MRSA colonization of wounds. Of 19 patients whose wounds were colonized with MRSA, 13 had persistent or intermittent colonization of these wounds even though the nares had been cleared of MRSA. In the second 5 months, wound MRSA colonization data were available on a weekly basis and showed that MRSA was cleared rapidly from wounds. Fourteen of 17 patients who began mupirocin in the second 5 months had wounds colonized with MRSA. In all but 2 of these 14 patients (14.3\%), MRSA was eliminated from their wounds after 1 week of therapy; 1 patient, who had an ischemic ulcer, remained positive after 2 weeks of therapy. Fifty percent of patients whose wounds were treated had ischemic ulcers, $23 \%$ had 


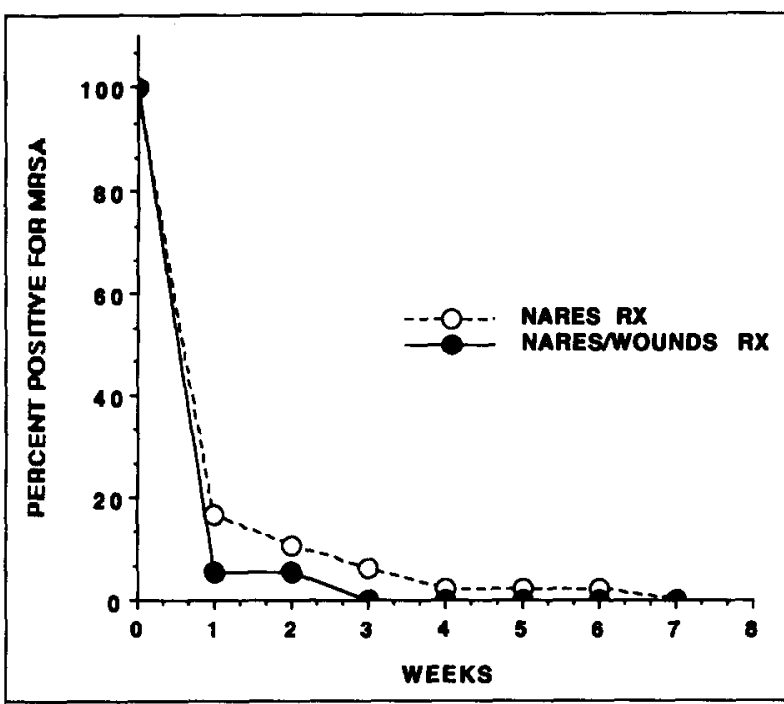

Figure 1. Eradication of MRSA in anterior nares in patients treated with mupirocin ointment applied only to anterior nares (open circles with broken line) or to both anterior nares and wounds (solid circles with solid line).

\section{TABLE I}

Colonization With Methicillin-Resistant S. aureus (MRSA) at Various Sites Before and After Treatment With Mupirocin*

\begin{tabular}{|c|c|c|c|c|}
\hline \multirow[b]{2}{*}{$\begin{array}{l}\text { Sites } \\
\text { Sampled }\end{array}$} & \multirow{2}{*}{$\begin{array}{c}\text { Prior to } \\
\text { Mupirocin } \\
\text { Use } \\
(6 / 89-6 / 90)\end{array}$} & \multicolumn{2}{|c|}{ Mupirocin Treatment } & \multirow[b]{2}{*}{ value } \\
\hline & & $\begin{array}{c}\text { Nares Only } \\
(6 / 90-12 / 90)\end{array}$ & $\begin{array}{l}\text { Nares/Wounds } \\
(1 / 91-6 / 91)\end{array}$ & \\
\hline $\begin{array}{l}\text { Nares } \\
\text { Perineum } \\
\text { Rectum } \\
\text { Wounds }\end{array}$ & $\begin{array}{r}15.2 \pm 1.0 \\
3.7 \pm 0.8 \\
4.6 \pm 0.9 \\
40.1 \pm 4.4\end{array}$ & $\begin{array}{r}12.0 \pm 1.6 \\
3.7 \pm 1.5 \\
5.3 \pm 0.6 \\
35.6 \pm 2.6\end{array}$ & $\begin{array}{r}6.3 \pm 0.8 \\
1.2 \pm 0.4 \\
1.4 \pm 0.5 \\
22.9 \pm 5.0\end{array}$ & $\begin{array}{l}0.0001 \\
0.260 \\
0.032 \\
0.057\end{array}$ \\
\hline
\end{tabular}

*Data are expressed as the mean percent of patients \pm SE colonized with MRSA each month at the sites indicated. Data analyzed by one-way analysis of variance.

texpressed as the percent of patients who had wounds that were colonized with MRSA

decubitus ulcers, $18 \%$ had abdominal or thoracic postoperative wounds, and $9 \%$ had tracheostomies.

Recurrence of MRSA in Patients Receiving Mupirocin

Recurrence of MRSA colonization in treated patients was not uncommon. During the first 7 months, 18 of $48(37.5 \%)$ patients had at least 1 recurrence of MRSA in the anterior nares. In the second 5 months, 8 of $17(47.1 \%)$ patients admitted into the study in the second 5 months and 1 of 10 (10\%) patients carried over from the first 7 months had a recurrence, overall rate $=9$ of $27(33.3 \%)$. Although most patients [14] had only one discrete episode of recurrence, eight patients had two, three patients had three, and one patient each had four and five recurrences. In 23 of 27 patients, recurrences were in nares, and in 4 patients recurrences were in wounds. However, wound data only include

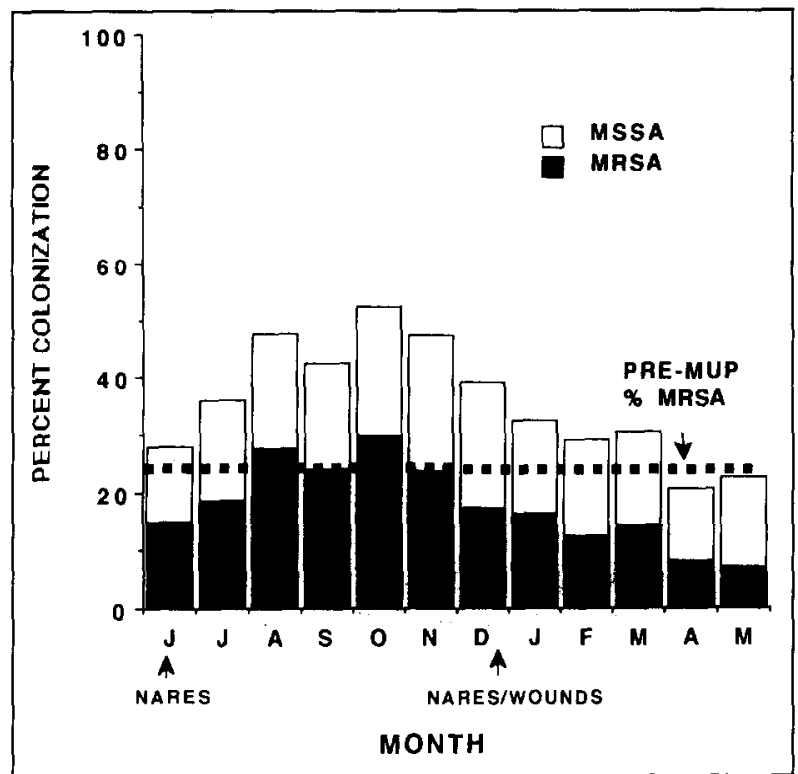

Flgure 2. Percent of patients colonized with MSSA (white bars) or MRSA (black bars) each month. The mean colonization rate for MRSA in the 12 months preceding use of mupirocin is shown as a broken line labeled "PRE-MUP \% MRSA." The time point at which mupirocin use was initiated for anterior nares only is indicated by the arrow labeled "Nares." The time point at which mupirocin was initiated for use in both anterior nares and wounds is indicated by the arrow labeled "Nares/Wounds." There was a significant difference in MRSA colonization when premupirocin use was compared with nares-only use and nares/wounds use, $p=0.0001$, by one-way analysis of variance.

those patients with MRSA colonization of wounds in the last 5 months of the study.

Of 48 separate episodes of recurrence, 46 of the MRSA strains were phage-typed; 20 (43.5\%) were a new phage group and presumably represented recolonization with a different organism; 26 (56.5\%) were the same phage group and most likely represented relapse with the original colonizing strain. All but 11 of these 48 episodes required an increase in the frequency of mupirocin administration to clear the organism. All recurrences but 2 occurred when the patient was receiving weekly maintenance mupirocin treatment.

\section{MRSA Colonization in the Facility}

Prior to the use of mupirocin (June 1, 1989, to June 1,1990$)$, the mean monthly colonization rate for MRSA in all residents of the facility was $22.7 \% \pm$ $1.0 \%$. This did not change during the 7 months mupirocin was used only in anterior nares $(22.2 \% \pm$ $2.1 \%$ ), but decreased significantly when both nares and wounds were treated $(11.5 \% \pm 1.8 \%), p=0.0001$ (Figure 2). By the last 2 months of the study, the MRSA colonization rate had fallen to $7.3 \%$. 
Flgure 3. Persistence of MRSA expressed as mean number of patients colonized each month with MRSA ( \pm SE), comparing the 6 months in which mupirocin was used in anterior nares only (Nares) with the 6 months in which mupirocin was used in both anterior nares and wounds (Nares/Wounds). Previously positive $(+)$ patients are those who were colonized with MRSA prior to mupirocin use in the facility and those who had a recurrence despite mupirocin use; $(t)$ on admission are patients who came into the facility with MRSA; new converters are patients who acquired MRSA in the facility. Data analyzed by one-way analysis of variance.

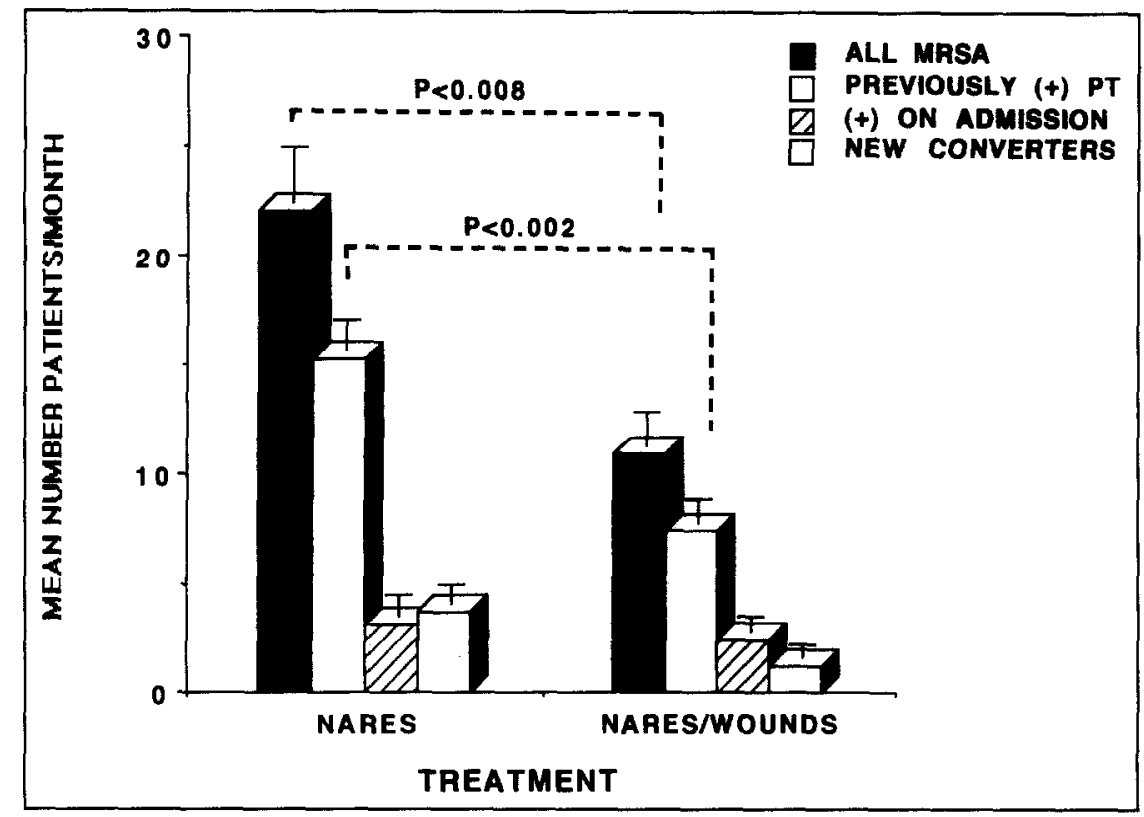

The overall rate of colonization with MSSA decreased slightly, comparing premupirocin colonization rates $(19.8 \% \pm 1.2 \%)$ with the first 7 months $(19.5 \% \pm 1.4 \%$ colonized) and the next 5 months of mupirocin use $(15.6 \% \pm 0.9 \%$ colonized), but these differences were not significant, $p=0.113$. Again, as with MRSA, the lowest MSSA colonization rate was noted in the last 2 months of the study.

Colonization rates at various sites for the year prior to mupirocin use, the 7 months when nares alone were treated, and the 5 months when nares and wounds were treated are shown in Table I. Colonization in all sites decreased with mupirocin use, but only when both nares and wounds were treated.

The persistence of MRSA in the facility was due to several factors (Figure 3). The number of new patients admitted to the facility already colonized with MRSA did not decrease over the course of the year. Recurrences of colonization during maintenance mupirocin treatment decreased in the second 5 months, but still continued to occur. New converters did decrease in the second 5 months of the study, but the decrease was not significant.

\section{Transmission of MRSA in the Facility}

Among all patients in the facility, acquisition of MRSA of the same phage group that was carried by a roommate was noted in eight patients in the first 7 months of the study, giving an incidence of transmission of $4 \%$. This number is similar to that noted in the prior year when nine patients $(3 \%)$ acquired MRSA of the same phage group noted in a roommate. When mupirocin was used in both anterior nares and wounds in the next 5 months, no roommate-to-roommate spread was documented.

\section{MRSA Infections in the Facility}

Only 6 of 321 patients developed infection with MRSA over the course of the year, 3 in the first 7 months and 3 in the next 5 months. Of these six patients, only three were known to be colonized with MRSA; two of these three had received treatment with mupirocin ointment for 2 weeks and 8 weeks prior to infection, and the third patient had refused mupirocin treatment. There were three patients with osteomyelitis, two with skin and soft tissue infections, one of whom was bacteremic, and one with a urinary tract infection. Five patients required hospitalization for intravenous vancomycin therapy; none died of their infection.

This rate of development of infection (6 of 321 patients, $1.9 \%$ ) did not differ from that noted in the prior year of the study when 9 of 341 patients (2.6\%) developed infection with MRSA while in the longterm-care facility.

\section{MRSA Contamination of the Environment}

At the start of the study, before mupirocin was used, $33(9 \%)$ of 380 samples from the environment were positive for MRSA. This decreased to 5.3\% (26 of $\mathbf{4 8 8}$ sites sampled) after the first 7 months and $0.6 \%$ ( 3 of 488 sites) by the end of the study, $\chi^{2}=$ $34.28, p=0.0001$. Floors by the patients' beds gave the highest yield of cultures positive for MRSA in the first two samplings, $39 \%$ and $42 \%$ of all isolates, respectively. Bedside tables were next most commonly positive for MRSA. Common resource areas 
were infrequently found to be contaminated with MRSA, with one environmental sample positive in June 1990, four positive in January 1991, and one positive in June 1991.

\section{Development of Resistance to Mupirocin}

Of those patients treated with mupirocin, 7 of 65 (10.8\%) had 8 mupirocin-resistant MRSA strains isolated. Seven of these 8 strains showed low-level resistance to mupirocin (MIC 3.1 to $62.5 \mu \mathrm{g} / \mathrm{mL}$ ) and 1 exhibited high-level mupirocin resistance (MIC greater than 5,000 $\mu \mathrm{g} / \mathrm{mL}$ ). Only one patient was colonized with a mupirocin-resistant strain at the time of admission to the facility, but it was later discovered that he had previously been treated with mupirocin at another facility. In five patients, the mupirocin-resistant strain was the same phage group as the prior colonizing strain, which was mupirocin-susceptible; two patients acquired a mupirocin-resistant strain that was a different phage group, and one isolate was not typed. Mupirocinresistant organisms appeared in nares as well as wounds; four strains appeared in the first 7 months and four in the second 5 months of the study. All patients colonized with low-level mupirocin-resistant strains cleared their organism with continued mupirocin treatment. The patient with the highlevel mupirocin-resistant MRSA isolate in his wound remained persistently colonized despite treatment with mupirocin. Neither he nor the other six patients developed infection with a mupirocinresistant MRSA strain.

In addition to the eight mupirocin-resistant MRSA isolates, four mupirocin-resistant MSSA strains were also isolated over the course of the year; all showed low-level resistance only. Only one mupirocin-resistant strain (a low-level resistant MSSA strain) was obtained from the environmental cultures.

\section{COMMENTS}

We have described the effects of long-term mupirocin use on both individual carriage of MRSA and overall colonization and infection rates within a long-term-care facility in which MRSA is endemic. We found that MRSA colonization of anterior nares alone or both anterior nares and wounds was quickly eradicated by mupirocin. Within a week, elimination of MRSA had occurred in most patients who were treated. This immediate effect is similar to prior findings reported for $S$. aureus in our longterm-care facility [13], in hospitalized patients [11], and in patients undergoing long-term hemodialysis [19]. When mupirocin was used in health care workers with nares and/or hand colonization, similar ef- ficacy for eradication of $S$. aureus has been described [14].

We found that monthly MRSA colonization rates in the long-term-care facility fell when mupirocin was applied to both nares and wounds of MRSA carriers. Similarly, environmental contamination with MRSA also decreased when wounds as well as nares were treated. However, we were unable to show a positive benefit of the use of mupirocin on MRSA infection rates. Most likely this is due to the small number of infections seen in our facility prior to the use of mupirocin [7]. On the other hand, in an outbreak situation with a high rate of MRSA infections, decolonization measures employing mupirocin are more likely to be beneficial in decreasing the infection rate $[11,20]$. In addition, two groups have documented a decrease in $S$. aureus infections in hemodialysis patients after initiation of the use of mupirocin [21,22].

In outbreaks, mupirocin ointment, often combined with other measures, has been found to be efficacious when used for a short period of time $[11,20,23]$. However, in the endemic setting, longterm use of mupirocin may be necessary. In a prior study from our long-term-care facility, follow-up cultures at 1 and 2 months after 7 days of mupirocin therapy showed that $44 \%$ of patients had a recurrence of $S$. aureus in their nares [13]. Most likely, the continuous introduction of new carriers of MRSA into the long-term-care setting obviates the benefit of short-term therapy with mupirocin. We sought to determine the minimum maintenance therapy required to prevent recurrences, and found that once-weekly application was not adequate to prevent breakthrough. In a different clinical situation, that of a hemodialysis unit, Boelaert et al [19] reported that once-weekly mupirocin was adequate to prevent relapse. However, in the hemodialysis unit, cultures were only performed monthly and may have missed transient breakthroughs.

We found that wounds, as well as nares, had to be treated to eradicate MRSA from wounds and to show an impact on overall colonization rates in the facility. This differs from findings in health care workers, in whom nasal application eliminated $S$. aureus from hands as well as nares [14]. This probably reflects transient colonization with lower numbers of organisms on the hands of health care workers when compared with more heavily colonized wounds in patients.

It is possible that the decrease in colonization was not directly related to the use of mupirocin, but was due to other factors. For example, the knowledge that a patient was colonized might change behavior on the part of the health care workers, inducing 
them to change their practices. We think this is unlikely since we saw no changes in colonization rates in the first 7 months when the patients' MRSA status was also known by the health care workers. Also, colonization rates in the first year, when the patients' MRSA status was not shared with staff, were the same as that of the first 7 months of the second year of the study. Only when mupirocin was used in both nares and wounds did MRSA colonization rates fall.

No side effects of mupirocin treatment were found, as noted by others using the polyethylene glycol formulation [23-25] and the paraffin-based formulation [11,14]. It is important to note that none of the wounds we treated had a large surface area, as might be seen in a burn patient or in a patient with an extensive decubitus ulcer. In patients with large wounds, absorption of polyethylene glycol-containing agents, such as mupirocin, nitrofurazone, or dextranomer beads, could occur and prove harmful [25].

It must be emphasized that the results we found may not apply to all long-term-care settings. MRSA has tended to be more prevalent in tertiarycare university hospitals and veterans facilities [3]. The physical attachment of the long-term-care facility to the acute-care hospital, as well as the presence of house staff to care for patients, differentiates our long-term-care facility from other community-based facilities. The fact that most of the patients were men may also make our findings not directly applicable to community-based longterm-care facilities serving predominantly women.

When drugs are used over a long period of time in an attempt to decolonize carriers, a major concern is the selection of resistant organisms. This concern has come to fruition in prior studies aimed at eradication of $S$. aureus with ciprofloxacin, rifampin, clindamycin, and trimethoprim-sulfamethoxazole [26-28]. Strausbaugh et al [28] recently documented the ineffectiveness of a decolonization program in a Veterans Affairs long-term-care facility in which the emergence of MRSA strains resistant to rifampin and clindamycin was common.

We documented both low-level and high-level resistance to mupirocin in isolates from patients in our facility. Resistance to mupirocin has now been reported in hospitals in England [29-34] and the United States [24,35]. Most resistance has been low-level, and perhaps may be of little clinical importance since the concentration of mupirocin is so high when applied as an ointment $(20,000 \mu \mathrm{g} / \mathrm{mL})$ [10]. In fact, our patients with low-level resistance all had MRSA clearance with continued mupirocin use, as have other $S$. aureus carriers described pre- viously $[29,35]$. This type of resistance is inducible in vitro with stepwise increases in mupirocin concentration, and does not appear to be plasmid-mediated or transferable.

In contrast, high-level resistance is uncommon [29], is plasmid-mediated [31], and has been associated with clinical failure to eradicate $S$. aureus $[31,32]$. In hospitals in England that have monitored susceptibility to mupirocin over several years, the percentage of resistant isolates of $S$. aureus is increasing $[30,33,34]$. This increase appears to be related to widespread use of the drug, especially in dermatology wards. We have also noted an increase in the number of institutions in our region using mupirocin liberally in an attempt to eradicate MRSA colonization. The concerns of those observing the phenomenon of increasing mupirocin resistance are mirrored in our data, which show the emergence of resistance to mupirocin in association with chronic use in a long-term-care facility with a high endemic rate of MRSA colonization.

Although overall MRSA colonization was decreased in the facility, patients continued to be admitted with MRSA and recurrences occurred in many patients receiving maintenance mupirocin treatment. Tracking all patients colonized with MRSA and following all patients undergoing maintenance mupirocin therapy to ensure they had not had a recurrence required a half-time research assistant. The microbiologic resources required were considerably more than available for most longterm-care facilities. Taking into account the costs for labor and supplies required to monitor colonization, the lack of real benefit in decreasing infection rates, and the very significant drawback of selection of mupirocin-resistant organisms, we believe that mupirocin should not be used chronically in longterm-care facilities that have endemic MRSA. Reserving mupirocin as one of several measures to stop an outbreak is a more appropriate use of this drug.

\section{REFERENCES}

1. Peacock JE, Marsik FJ, Wenzel RP. Methicillin-resistant Staphylococcus aureus: introduction and spread within a hospital. Ann Intern Med 1980; 93 : 526-32.

2. Thompson RL, Cabezudo I, Wenzel RP. Epidemiology of nosocomial infections caused by methicillin-resistant Staphylococcus aureus. Ann Intern Med 1982; 97: 309-17.

3. Boyce JM. Methicillin-resistant Staphylococcus aureus. Detection, epidemiolOgy, and control measures. Infect Dis Clin North Am 1989; 3: 901-13.

4. Hsu CCS, Macaluso CP, Special L, Hubble RH. High rate of methicillin-resistance of Staphylococcus aureus isolated from hospitalized nursing home patients. Arch Intern Med 1988; 148: 569-70.

5. Muder RL, Brennen C, Wagener MM, et al. Methicillin-resistant staphylococcal colonization and infection in a long-term care facility. Ann Intern Med 1991; 114: 107-12.

6. Strausbaugh L, Jacobson C, Sewell DL, Potter S, Ward TT. Methicillin-resis- 
tant Staphylococcus aureus in extended care facilities: experiences in a Veterans Affairs nursing home and a review of the literature. Infect Control Hosp Epidemiol 1991; 12: 36-45.

7. Bradley SF, Terpenning MS, Ramsey MA, et al. Methicillin-resistant Staphylococcus aureus: colonization and infection in a long-term care facility. Ann intern Med 1991; 115: 417-22.

8. Boyce JM. Methicillin-resistant Staphylococcus aureus in nursing homes: putting the problem in perspective. Infect Control Hosp Epidemiol 1991; 12: 413-5. 9. Kauffman CA, Bradley SF, Terpenning MS. Methicillin-resistant Staphylococcus aureus in long-term care facilities. Infect Control Hosp Epidemiol 1990; 11: $600-3$.

10. Casewell MW, Hill RLR. Mupirocin (pseudomonic acid)-a promising new topical antimicrobial agent. J Antimicrob Chemother 1987; 19: 1-5.

11. Hill RLR, Duckworth GJ, Casewell MW. Elimination of nasal carriage of methicillin-resistant Staphylococcus aureus with mupirocin during a hospital outbreak. J Antimicrob Chemother 1988; 22: 377-84.

12. Hospital Infection Society and British Society for Antimicrobial Chemotherapy. Guldelines for the control of epldemic methicillin-resistant Staphylococcus aureus. J Hosp Infect 1986; 7: 193-201.

13. Cederna JE, Terpenning MS, Ensberg M, Bradley SF, Kauffman CA. Staphy lococcus aureus nasal colonization in a nursing home: eradication with mupirocin. Infect Control Hosp Epidemiol 1990; 11: 13-6.

14. Reagen DR, Doebbeling BN, Pfaller MA, et al. Elimination of coincident Staphylococcus aureus nasal and hand carriage with intranasal application of mupirocin calcium ointment. Ann Intern Med 1991; 114: 101-6.

15. Garner JS, Jarvis WR, Emori TG, Horan TC, Hughes JM. CDC definitions for nosocomial infections, 1988. Am J Infect Control 1988; 16: 128-40.

16. Thornsberry $C$, McDougal LK. Successful use of broth microdilution in susceptibility tests for methicillin-resistant (heteroresistant) staphylococci. J Clin Microbiol 1988; 16: 128-40.

17. Smith PB. Bacteriophage typing of Staphylococcus aureus. In: Cohen JO, editor. The staphylococci. New York: John Wiley \& Sons, 1992: 431-41.

18. Fuchs PC, Jones RN, Barry AL. Interpretive criteria for disk diffusion susceptibility testing of mupirocin, a topical antibiotic. J Clin Microbiol 1990; 28: 608-9.

19. Boelaert JR, Godard C, deBaere Y, Gordts 8 , van Landuyt HW. Once weekly nasal mupirocin in hemodialysis patients [abstract 341]. Program and abstracts of the 31 st Interscience Conference on Antimicrobial Agents and Chemotherapy, Chicago, 1991.

20. Barrett SP. The value of nasal mupirocin in containing an outbreak of methicillin-resistant Staphylococcus aureus in an orthopaedic unit. J Hosp Infect 1990; 15: 137-42.
21. Boelaert JR, deBaere $Y$, Godard C, van Landuyt HW. Eradication of Staphy lococcus aureus in dialysis by nasal mupirocin [abstract 1262]. Program and abstracts of the 29th Interscience Conference on Antimicrobial Agents and Chemotherapy, Houston, 1989.

22. Holton D, Nicolle LE, Diley D, Bernstein K. Efficacy of mupirocin nasal ointment in eradicating Staphylococcus aureus nasal carriage in chronic hemodialysis patients. J Hosp Infect $1991 ; 17$ : 133-7.

23. Darouiche R, Wright C, Hamill R, Koza M, Lewis D, Markowski J. Eradication of colonization by methicillin-resistant Staphylococcus aureus by using oral minocycline-rifampin and topical mupirocin. Antimicrob Agents Chemother 1991; 35: 1612-5

24. Reagen DR, Dula RT, Palmer BH, Gutierrez CN, Franzus BF, Sarubbi FA. Control of MRSA in a VAMC with limited resources [abstract 31]. Program and abstracts of the 31st Interscience Conference on Antimicrobial Agents and Chemotherapy. Chicago, 1991.

25. Rode H, Hanslo D, de Wet PM, Miller AJW, Cywes S. Efficacy of mupirocin in methicillin-resistant Staphylococcus aureus burn wound infection. Antimicrob Agents Chemather 1989; 33: 1358-61.

26. Mulligan ME, Ruane PJ, Johnston L, et al. Ciprofloxacin for eradication of methicillin-resistant Staphylococcus aureus colonization. Am J Med 1987; 82 Suppl 4A: 215-9.

27. Peterson LR, Quick JN, Jensen B, et al. Emergence of ciprofloxacin resistance in nosocomial methicillin-resistant Staphylococcus aureus isolates. Arch Intern Med 1990; 150: 2151-5.

28. Strausbaugh $L$, Jacobson $C$, Sewell $D L$, Potter $S$, Ward $\pi$. Antimicrobial therapy for methicillin-resistant Staphylococcus aureus colonization in residents and staff of a Veterans Affairs nursing home care unit. Infect Control Hosp Epidemiol 1982; 13: 151-9

29. Cookson BD. Mupirocin resistance in staphylococci. J Antimicrob Chemother 1990; 25: 497-503.

30. Wise R, Johnson J. Mupirocin resistance [letter]. Lancet 1991; 338: 578. 31. Rahman M, Noble WC, Cookson B. Transmissible mupirocin resistance in Staphylococcus aureus. Epidemiol Infect 1989; 102: 261-70.

32. Smith GE, Kennedy CTC. Staphylococcus aureus resistant to mupirocin [letter]. J Antimicrob Chemother 1988; 21: 141-2.

33. Kavi J, Andrews JM, Wise R. Mupirocin-resistant Staphylococcus aureus [letter]. Lancet 1987; 2: 1472.

34. Baird D, Coia J. Mupirocin-resistant Staphylococcus aureus [letter]. Lancet 1987; 2: 387-8.

35. Scully BE, Briones F, Gu J-w, Neu HC. Mupirocin treatment of nasal staphylococcal colonization. Arch Intern Med 1992; 152: 353-6. 